This is a pre-print of an article published in Journal of Materials Science: Materials in Electronics. The final authenticated version is available online at: https://doi.org/10.1007/s10854-017-8265-8

\title{
Defect Formation in Supported Graphene Irradiated by Accelerated Xenon Ions
}

Egor A. Kolesov ${ }^{1}$, Mikhail S. Tivanov ${ }^{1}{ }^{*}$, Olga V. Korolik ${ }^{1}$, Pavel Yu. Apel ${ }^{2,}{ }^{3}$, Vladimir A. Skuratov $^{2,3,4}$, Anis Saad $^{5}$, Ivan V. Komissarov ${ }^{6}$

${ }^{1}$ Belarusian State University, 4 Nezavisimosti Av., 220030 Minsk, Belarus

${ }^{2}$ Joint Institute for Nuclear Research, Joliot-Curie 6, 141980 Dubna, Russia

${ }^{3}$ Dubna State University, Dubna, Russia

${ }^{4}$ National Research Nuclear University MEPhI, Moscow, Russia

${ }^{5}$ Al-Balqa Applied University, PO Box 4545, Amman 11953, Jordan

${ }^{6}$ Belarusian State University of Informatics and Radioelectronics, 6 P. Brovka, 220013 Minsk, Belarus

* Corresponding author: E-mail tivanov@ bsu.by; Phone +375172095451; Fax +375172095445; Address: 4 Nezavisimosti Av., 220030 Minsk, Belarus.

\section{Abstract}

Raman spectroscopy and Monte-Carlo simulation studies for supported graphene irradiated by $160 \mathrm{MeV} \mathrm{Xe}$ ions are presented. Changes in the density and dominating types of defects with increasing fluence were observed. In order to analyze contribution of defect formation mechanisms, in which the substrate is involved, a comparative study was performed for graphene on $\mathrm{SiO}_{2} / \mathrm{Si}$, copper and glass substrates. The major defining mechanisms were found to be 
atomic recoils and formation of defects induced by hot electrons. For graphene on copper, the impact of substrate recoil atoms was found to be greater comparing to graphene on silicon oxide and glass, where the recoils participated approximately equally. Moreover, a possibility of defect formation in graphene due to hot electrons generated in the substrate near the interface was noted. Finally, a linear dependence of air-induced doping on D and G peak intensity ratio that represents defect density in graphene was found. The study is useful for solving the longstanding controversy on major mechanisms of defect formation in irradiated graphene, as well as for graphene-based nanoelectronic device engineering.

Keywords: graphene; irradiation; swift heavy ions; interface; TRIM; adsorption.

\section{Introduction}

Graphene is a promising material for a variety of applications due to its unique physical properties [1]. At present, graphene is used in transparent electrodes, field effect transistors, sensors and other applications. Engineering of several graphene-based nanoelectronic devices such as biosensors, as well as graphene functionalization techniques, requires structural modification of the material through a controlled defect introduction [2-4]. Swift heavy ions (SHI) irradiation method is a versatile and convenient tool for this purpose [5]. It allows one to control defect densities varying type, energy and fluence of ions. However, the mechanism of defect formation in SHI-irradiated graphene still requires additional clarification.

It is known that the events of energy transfer to both target lattice nuclei (producing recoils) and electron sub-system (producing hot electrons) participate in the ion-matter interaction process, the latter giving non-negligible contribution to the defect formation in graphene [6]. Moreover, the substrate was ambiguously reported to affect graphene stability under the irradiation, leading either to creation of additional defects [7, 8] or to reduction of the resultant defect yield [9].

Raman spectroscopy is a versatile tool for obtaining information on the structural properties of various materials. This method is particularly useful for graphene studies due to 
monoatomic thickness of the material [1]. Purpose of the present work is to study defect formation processes in SHI-irradiated supported graphene using Raman spectroscopy and Monte-Carlo TRIM simulations in order to understand the contributions of different defect formation mechanisms.

\section{Experimental}

Graphene was obtained using an atmospheric-pressure chemical vapor deposition (CVD). Prior to the synthesis, copper substrate was electrochemically polished in $1 \mathrm{M}$ phosphoric acid solution for $5 \mathrm{~min}$ with an operating voltage of $2.3 \mathrm{~V}$. The CVD process was performed in a tubular quartz reactor with a diameter of $14 \mathrm{~mm}$ using reagent grade chemicals. Copper foil was pre-annealed at $1050{ }^{\circ} \mathrm{C}$ for 60 min under the following gas flow rates: $\mathrm{H}_{2}-150 \mathrm{cc} / \mathrm{min}, \mathrm{N}_{2}-$ $100 \mathrm{cc} / \mathrm{min}$. The synthesis was performed under the following conditions: reactor temperature of $1050{ }^{\circ} \mathrm{C}, \mathrm{C}_{10} \mathrm{H}_{22}$ (decane) flow rate of $4 \mu \mathrm{l} / \mathrm{min}, \mathrm{H}_{2}$ flow rate of $60 \mathrm{cc} / \mathrm{min}, \mathrm{N}_{2}$ carrier flow rate of $100 \mathrm{cc} / \mathrm{min}$, and synthesis time of $30 \mathrm{~min}$. After the hydrocarbon flow had been terminated, the sample was cooled down to room temperature at a rate of $\sim 50{ }^{\circ} \mathrm{C} / \mathrm{min}$.

Graphene was transferred to $\mathrm{SiO}_{2} / \mathrm{Si}$ (oxide thickness of $600 \mathrm{~nm}$ ) and glass substrates by a wet-chemical room-temperature etching without polymer support in two steps. First, one side of copper foil was treated for 3 min in a solution of $\mathrm{H}_{2} \mathrm{NO}_{3}$ and $\mathrm{H}_{2} \mathrm{O}$ mixed in a volume ratio of 1:3. Then, the copper foil was totally dissolved in a water solution of $\mathrm{FeCl}_{3}$. Graphene film was washed several times in a distilled water bath prior to being placed onto the substrate.

Graphene was irradiated by $160 \mathrm{MeV}$ xenon ions with fluences of $10^{8}, 10^{9}$ and $10^{11} \mathrm{~cm}^{-2}$ at the IC100 cyclotron at the FLNR JINR in Dubna [10]. Ion beam homogeneity over the irradiated sample surface was controlled using beam scanning in the horizontal and vertical directions and was better than $5 \%$.

Raman spectra were obtained with a spectral resolution better than $3 \mathrm{~cm}^{-1}$ using a Nanofinder HE (LOTIS TII) confocal Raman spectrometer. For excitation of Raman radiation, a continuous solid-state laser with a wavelength of $473 \mathrm{~nm}$ was used. Room-temperature Raman 
measurements were carried out using laser power of $800 \mu \mathrm{W}$, the diameter of laser spot on the sample surface being of about $0.6 \mu \mathrm{m}$.

The simulations were performed using binary collision Monte-Carlo approach implemented in TRIM (the Transport of Ions in Matter) code [11]. TRIM group of programs uses quantum-mechanical collision treatment considering screened Coulomb interaction between an ion with effective charge and an atom, including exchange and correlation interactions for the overlapping electron shells and creation of electron excitations and plasmons inside the target [11]. In order to estimate the role of various defect formation mechanisms in the evolution of graphene-substrate system under ion irradiation, the Monolayer Collision mode (for every collision to be calculated without any approximations) with 100,000 incident $160 \mathrm{MeV}$ xenon ions was utilized for graphene monolayer simulated located on a substrate layer with the thickness of $300 \AA$.

It is important to note that TRIM code treats target as an amorphous matrix with a homogenous mass distribution (which is not generally applicable for nanostructures) and calculates each collision impact regardless of collision density. Thus, TRIM simulation of graphene irradiation cannot be used in order to obtain specific quantitative results. However, TRIM code can still be used for statistical qualitative estimations [12-14]. Besides, supported graphene can be considered a bulk system within the scale of several effects such as substrate sputtering. Thus, we preferred TRIM code for the simulation since we were interested in a statistical description of various defect formation mechanisms attributed to the substrate as a bulk system participating in the process.

\section{Results and discussion}

Typical Raman spectra of pristine and $\mathrm{SHI}$-irradiated graphene on $\mathrm{SiO}_{2} / \mathrm{Si}$ substrate are presented in Fig. 1. As seen, D peak in pristine graphene spectrum has relatively low intensity $\left(\mathrm{I}_{\mathrm{D}} / \mathrm{I}_{\mathrm{G}} \sim 0.18\right)$, which corresponds to a small disorder of the sample structure $[1,15]$. At the same time, Raman spectra of irradiated graphene demonstrate increase in $\mathrm{I}_{\mathrm{D}} / \mathrm{I}_{\mathrm{G}}$ intensity ratio of up to 
0.5 (in several points - up to 1.0) as the irradiation fluence changes from $10^{8}$ to $10^{11} \mathrm{~cm}^{-2}$, indicating an increase of defect concentration. Moreover, position shift of the second-order 2D peak from 2687 to $2691 \mathrm{~cm}^{-1}$, the decrease of $\mathrm{I}_{2 \mathrm{D}} / \mathrm{I}_{\mathrm{G}}$ intensity ratio from 1.7 to 1.0 , and the increase of $2 \mathrm{D}$ peak width from 33 to $43 \mathrm{~cm}^{-1}$ are observed. These changes together with the shift of $\mathrm{G}$ peak position from 1587 to $1595 \mathrm{~cm}^{-1}$ can be attributed to adsorption doping of airexposed defected graphene and are discussed later.

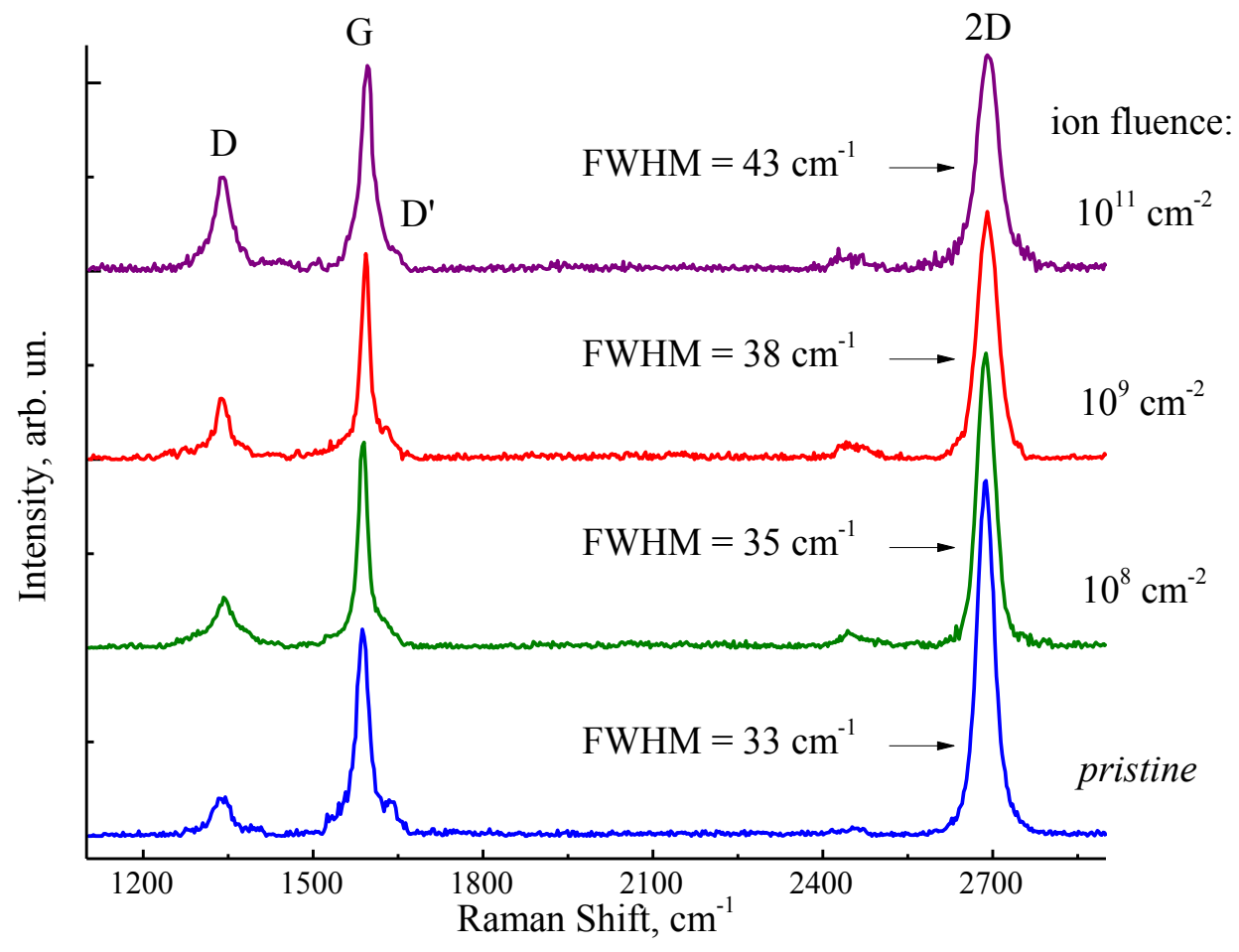

Figure 1. Typical Raman spectra of pristine and $\mathrm{SHI}$-irradiated graphene on $\mathrm{SiO}_{2} / \mathrm{Si}$ substrate. Irradiation fluence is given to the right of the plots.

In order to obtain information on defect distribution over the sample surfaces, Raman mappings of $20 \times 20 \mu \mathrm{m}^{2}$ areas were obtained (Fig. 2). A uniform increase of $\mathrm{I}_{\mathrm{D}} / \mathrm{I}_{\mathrm{G}}$ intensity ratios (and therefore, the defect densities) is observed with increasing fluence. Raman map of graphene irradiated with the fluence of $10^{11} \mathrm{~cm}^{-2}$ also demonstrates presence of high defect density regions, possibly corresponding to a partial destruction of graphene lattice. 

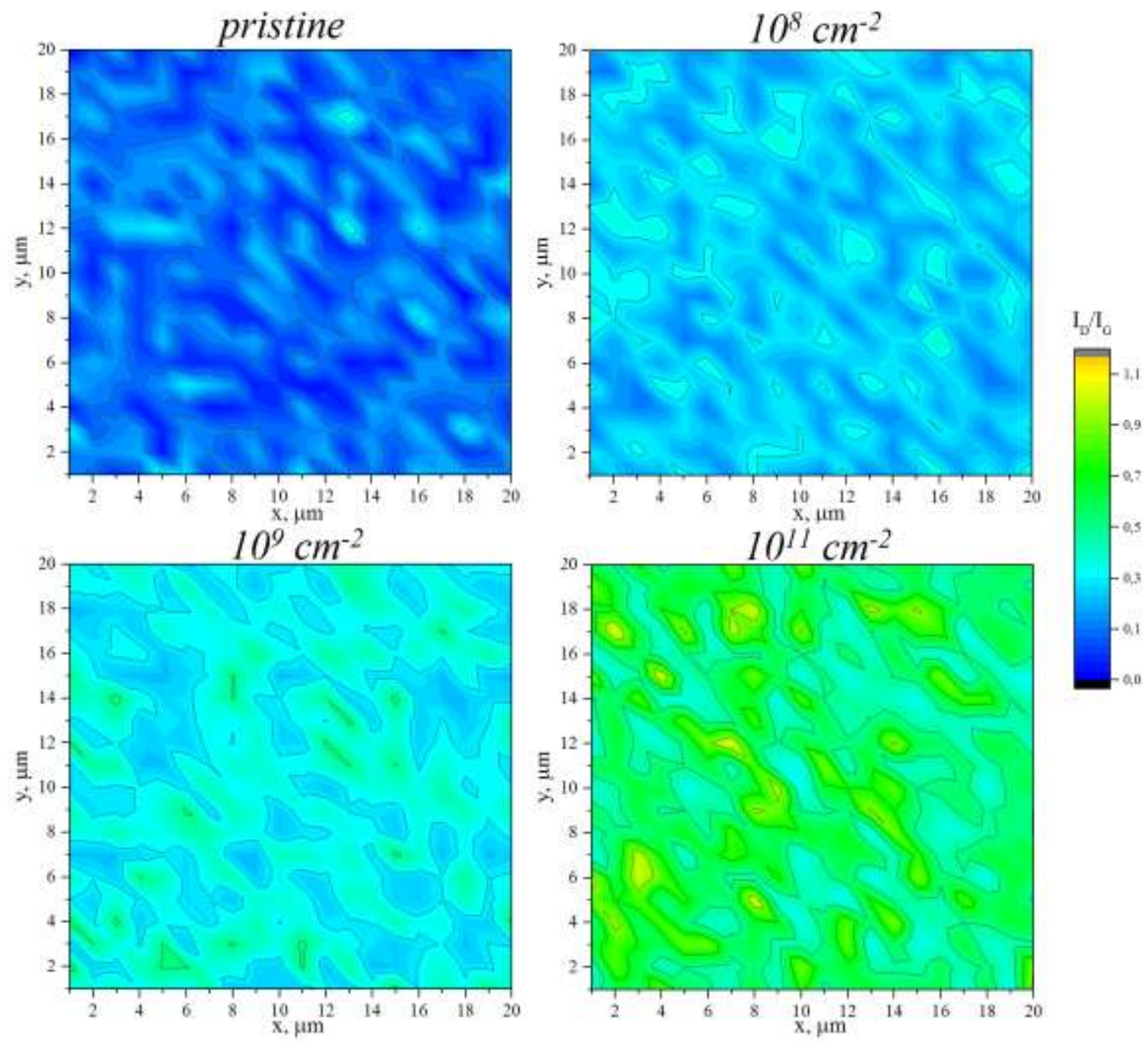

Figure 2. Raman maps $\left(20 \times 20 \mu \mathrm{m}^{2}\right)$ of $\mathrm{I}_{\mathrm{D}} / \mathrm{I}_{\mathrm{G}}$ intensity ratio representing defect density distribution over the sample surface for pristine and irradiated graphene on $\mathrm{SiO}_{2} / \mathrm{Si}$ substrate; scanning step of $1 \mu \mathrm{m}$.

Raman process leading to $\mathrm{D}$ peak arising in the spectrum implies relaxation of zerowavevector selection rule and includes events of elastic scattering on a defect and inelastic scattering on $\mathrm{A}_{1 \mathrm{~g}}$ phonon at the Brillouin zone edge [15]; therefore, $\mathrm{I}_{\mathrm{D}} / \mathrm{I}_{\mathrm{G}}$ ratio is directly related to the defect density. This dependence includes regions of inverse proportionality (high density of defects) and direct proportionality (low-density region) [15]. However, the former means the disorder is large enough to affect $G$ and 2D peak profiles [15], and that was not observed in the experimental spectra. Thus, we assume that the performed irradiation introduced degree of disorder specific for a low-density region, and therefore $\mathrm{I}_{\mathrm{D}} / \mathrm{I}_{\mathrm{G}}$ is directly proportional to the defect density, which is confirmed by the relationship between the experimental fluence and 
$\mathrm{I}_{\mathrm{D}} / \mathrm{I}_{\mathrm{G}}$ values. It is also important to note that since the $\mathrm{I}_{\mathrm{D}} / \mathrm{I}_{\mathrm{G}}$ ratio does not depend on a defect geometry [16], its values represent the average interdefect distance as well as corresponding density for all Raman active defect sites that are involved in elastic scattering events.

As it was shown in [16], information on dominating defect type can be provided by $\mathrm{I}_{\mathrm{D}} / \mathrm{I}_{\mathrm{D}}$, intensity ratio in Raman spectra of graphene. The average $\mathrm{I}_{\mathrm{D}} / \mathrm{I}_{\mathrm{D}}$, values obtained from the maps are presented in Table 1. Raman spectra of pristine graphene demonstrate $\left\langle\mathrm{I}_{\mathrm{D}} / \mathrm{I}_{\mathrm{D}}\right.$, $\rangle$ values of about 1.5, corresponding to grain boundaries as dominating defects [16]. At the same time, Raman spectroscopy data for the irradiated graphene shows $\left\langle\mathrm{I}_{\mathrm{D}} / \mathrm{I}_{\mathrm{D}}\right\rangle \sim 4.1-7.5$. According to dependencies presented in [16], these values indicate presence of both grain boundaries and vacancies, with the role of vacancies increasing for greater fluences (Table 1).

Table 1. Peak intensity ratios calculated from the Raman spectra of pristine and irradiated graphene on $\mathrm{SiO}_{2} / \mathrm{Si}$.

\begin{tabular}{|c|c|c|c|c|}
\hline Irradiation fluence, $\mathrm{cm}^{-2}$ & 0 (pristine) & $1 \cdot 10^{8}$ & $1 \cdot 10^{y}$ & $1 \cdot 10^{11}$ \\
\hline$<\mathrm{I}_{\mathrm{D}} / \mathrm{I}_{\mathrm{G}}>$ & 0.16 & 0.29 & 0.41 & 0.62 \\
\hline$<\mathrm{I}_{\mathrm{D}} / \mathrm{I}_{\mathrm{D}}>>$ & 1.5 & 4.1 & 5.2 & 7.5 \\
\hline
\end{tabular}

Fig. 3 demonstrates Raman maps of $\mathrm{I}_{\mathrm{D}} / \mathrm{I}_{\mathrm{D}}$, intensity ratio representing the distribution of different types of defects over the sample surface for pristine and irradiated graphene. As seen, grain boundaries are mostly typical for pristine graphene, with several regions demonstrating $\mathrm{I}_{\mathrm{D}} / \mathrm{I}_{\mathrm{D}}$, values corresponding to $\mathrm{sp}^{3}$-bonding complexes. The map for graphene irradiated with $10^{8}$ $\mathrm{cm}^{-2}$ fluence clearly shows the uniform increase of $\mathrm{I}_{\mathrm{D}} / \mathrm{I}_{\mathrm{D}}$, ratio, indicating the formation of irradiation-induced vacancies. As the fluence increases, further growth of vacancy density, as well as formation of more $\mathrm{sp}^{3}$-bonding complexes are observed. 

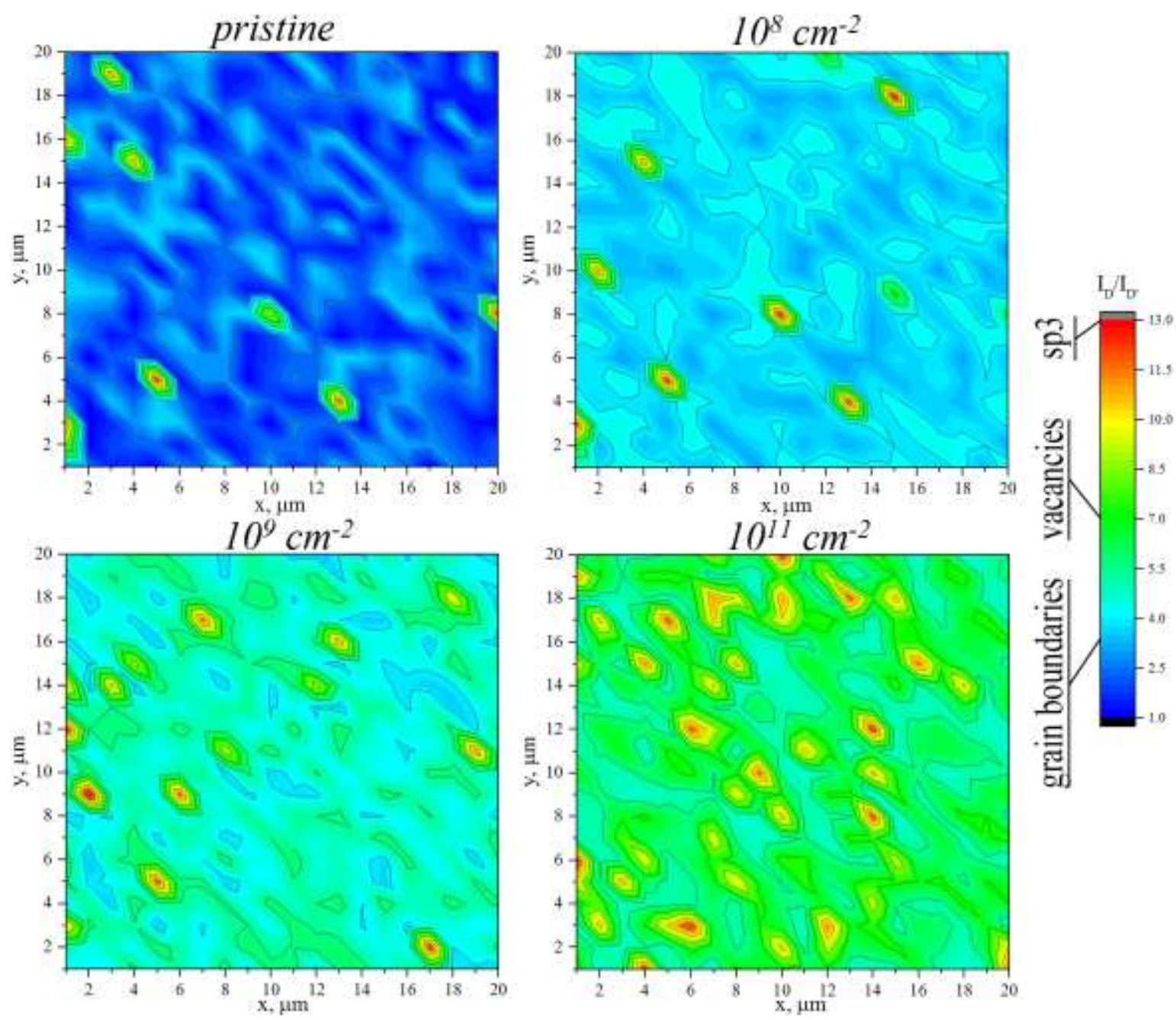

Figure 3. Raman maps $\left(20 \times 20 \mu \mathrm{m}^{2}\right)$ of $\mathrm{I}_{\mathrm{D}} / \mathrm{I}_{\mathrm{D}}$, intensity ratio representing defect type distribution over the sample surface for pristine and irradiated graphene on $\mathrm{SiO}_{2} / \mathrm{Si}$ substrate; scanning step of $1 \mu \mathrm{m}$. The types of dominating defects corresponding to specific $\mathrm{I}_{\mathrm{D}} / \mathrm{I}_{\mathrm{D}}$, values are indicated to the left of the color scale.

The observed $\mathrm{I}_{\mathrm{D}} / \mathrm{I}_{\mathrm{G}}$ increase is stronger than expected considering literature data for various ion types and energies, including data for suspended graphene [7, 17-19]. At the same time, it was reported that defect yields for suspended and supported graphene can differ significantly since the substrate can play an important role in defect formation process $[7,8,17,18,20]$. Particularly, the substrate might be involved through various different events such as energy transfer from a recoiled substrate atom to graphene lattice. Moreover, one cannot exclude the possibility of defect formation mechanism dependence on the crystallinity of the substrate 
material. Thus, a correct analysis of the obtained results requires paying a close attention to irradiation-induced substrate effects.

According to [17], the defect formation process in SHI-irradiated graphene includes defects generated through an indirect process of substrate sputtering. In order to determine the role of sputtered substrate atoms in graphene irradiation effects, comparison was carried out for graphene irradiated with a fixed fluence of $10^{8} \mathrm{~cm}^{-2}$ on copper (as-synthesized), $\mathrm{SiO}_{2} / \mathrm{Si}$ and glass substrates. We performed TRIM simulations for graphene on each substrate in order to obtain specific values of substrate sputtering yields, sputtered atom energy distributions, nuclear and electronic stopping power (it should be noted that TRIM code is quantitatively applicable for this case, since it is a bulk target being sputtered). Based on these values as well as defect yield / nuclear stopping power dependencies from [17], we estimated approximate defect yields for sputtered atoms. As an additional verification of structural modification trends obtained during the simulation, Raman maps of pristine and irradiated graphene were scanned on each substrate, whereupon average $\left\langle\mathrm{I}_{\mathrm{D}} / \mathrm{I}_{\mathrm{G}}\right\rangle$ and $\left\langle\mathrm{I}_{\mathrm{D}} / \mathrm{I}_{\mathrm{D}}\right\rangle$ parameters were determined. The comparison of the obtained values for graphene on various substrates is given in Table 2. Naturally, the number of backscattered ions was negligibly small for all cases.

Table 2. Values calculated from Raman spectra of pristine and irradiated (fluence of $10^{8} \mathrm{~cm}^{-2}$ ) graphene on various substrates, as well as parameters obtained using TRIM simulations.

\begin{tabular}{|c|c|c|c|}
\hline Substrate & Copper & $\mathrm{SiO}_{2} / \mathrm{Si}$ & Glass \\
\hline Pristine $\left\langle\mathrm{I}_{\mathrm{D}} / \mathrm{I}_{\mathrm{G}}>\right.$ & 0.15 & 0.16 & 0.18 \\
\hline Irradiated $\left\langle\mathrm{I}_{\mathrm{D}} / \mathrm{I}_{\mathrm{G}}>\right.$ & 0.34 & 0.29 & 1.5 \\
\hline Pristine $\left\langle\mathrm{I}_{\mathrm{D}} / \mathrm{I}_{\mathrm{D}}>\right.$ & 1.4 & 1.5 & 3.1 \\
\hline Irradiated $\left\langle\mathrm{I}_{\mathrm{D}} / \mathrm{I}_{\mathrm{D}}>\right.$ & & 4.1 & 0.06 \\
\hline Nuclear stopping power $\mathrm{S}_{\mathrm{n}}, \mathrm{keV} / \mathrm{nm}$ & 3.7 & 0.06 & 14.6 \\
\hline Electronic stopping power $\mathrm{S}_{\mathrm{e}}, \mathrm{keV} / \mathrm{nm}$ & 0.19 & 14.5 & $0.001-0.009$ \\
\hline Sputtering yield $\mathrm{Y}_{\mathrm{s}}$ & 36.3 & $0.006-0.008$ & \\
\hline
\end{tabular}




\begin{tabular}{|c|c|c|c|}
\hline Sputtered atom energies, keV & 2.7 & $4.3-4.7$ & $0.2-8.1$ \\
\hline Estimated defect yields for sputtered atoms & 1.7 & $0.6-1.2$ & $0.2-1.0$ \\
\hline
\end{tabular}

Due to the fact that $\left\langle\mathrm{I}_{\mathrm{D}} / \mathrm{I}_{\mathrm{G}}\right\rangle$ value of 0.15 for pristine as-grown graphene is quite close to those of 0.16 and 0.18 for graphene transferred to $\mathrm{SiO}_{2} / \mathrm{Si}$ and glass, we can confirm that the transfer process introduced only a minor amount of defects into graphene lattice. It is seen that $\left.<\mathrm{I}_{\mathrm{D}} / \mathrm{I}_{\mathrm{G}}\right\rangle$ increase after graphene irradiation on $\mathrm{SiO}_{2} / \mathrm{Si}$ and glass has close values, while one for copper is slightly greater. At the same time, the evolution of the defect system in graphene with the irradiation has similar direction for all three substrates: from grain boundaries domination in pristine graphene to both grain boundaries and vacancies in the irradiated material. However, vacancy formation turned out to be the most discernible effect for graphene on silicon oxide, according to utilized Raman dependencies from [16].

Due to a high energy of the incident ions, the energy transferred to the substrate nuclei for all three materials is much smaller than that transferred to the electron sub-system, all the sputtering yields being less than one (for copper, however, the sputtering yield is almost two orders of magnitude greater). Performing simple estimations considering presented in Table 2 defect yields for sputtered atoms, sputtering yields and fluence, one can obtain the following approximate total maximum defect concentrations created by sputtered substrate atoms during the irradiation: $2.4 \cdot 10^{7} \mathrm{~cm}^{-2}$ for copper, $9.6 \cdot 10^{5} \mathrm{~cm}^{-2}$ for $\mathrm{SiO}_{2} / \mathrm{Si}$ and $9.0 \cdot 10^{5} \mathrm{~cm}^{-2}$ for glass. These values are by several orders of magnitude smaller than the typical intrinsic defect density for a pristine material; thus, greater obtained $\mathrm{Y}_{\mathrm{s}}$ value for copper still implies a weak participation in the defect generation process. Thus, we do not consider substrate sputtering to play a major role in the defect formation in graphene irradiated with $160 \mathrm{MeV}$ xenon ions: substrate sputtering yields have maxima at smaller incident ion energies (less than $1 \mathrm{MeV}$ ). Besides, other works demonstrate that the sputtering damage of graphene itself effectively takes place for incident ion energies as small as $20 \mathrm{eV}$ [21], while still occurring most actively for irradiation by heavy ions $[18,22]$. Thus, using high-energy ions (more than $\sim 30 \mathrm{MeV}$, according to our TRIM 
simulations) for controlled defect induction in supported graphene can minimize the amount of defects induced by substrate sputtering.

However, according to our simulation results, there is a non-negligible role of substrate recoil atoms that do not leave the sample but become moved away from their positions at this irradiation energy. Due to the energy transferred from the incident ions, such recoils can reach graphene-substrate interface and participate in the defect formation. Fig. 4 demonstrates spatial recoil distributions statistically obtained for graphene on copper, $\mathrm{SiO}_{2} / \mathrm{Si}$ and glass substrates as another important output of TRIM simulation procedures. The greatest amount of recoils reaching graphene-substrate interface region is observed for graphene on copper. For graphene supported by $\mathrm{SiO}_{2} / \mathrm{Si}$ and glass, almost similar situation is observed, with the amount of oxygen atoms reaching the interface being slightly greater; however, $\mathrm{Na}$ atoms also participate in the second case.
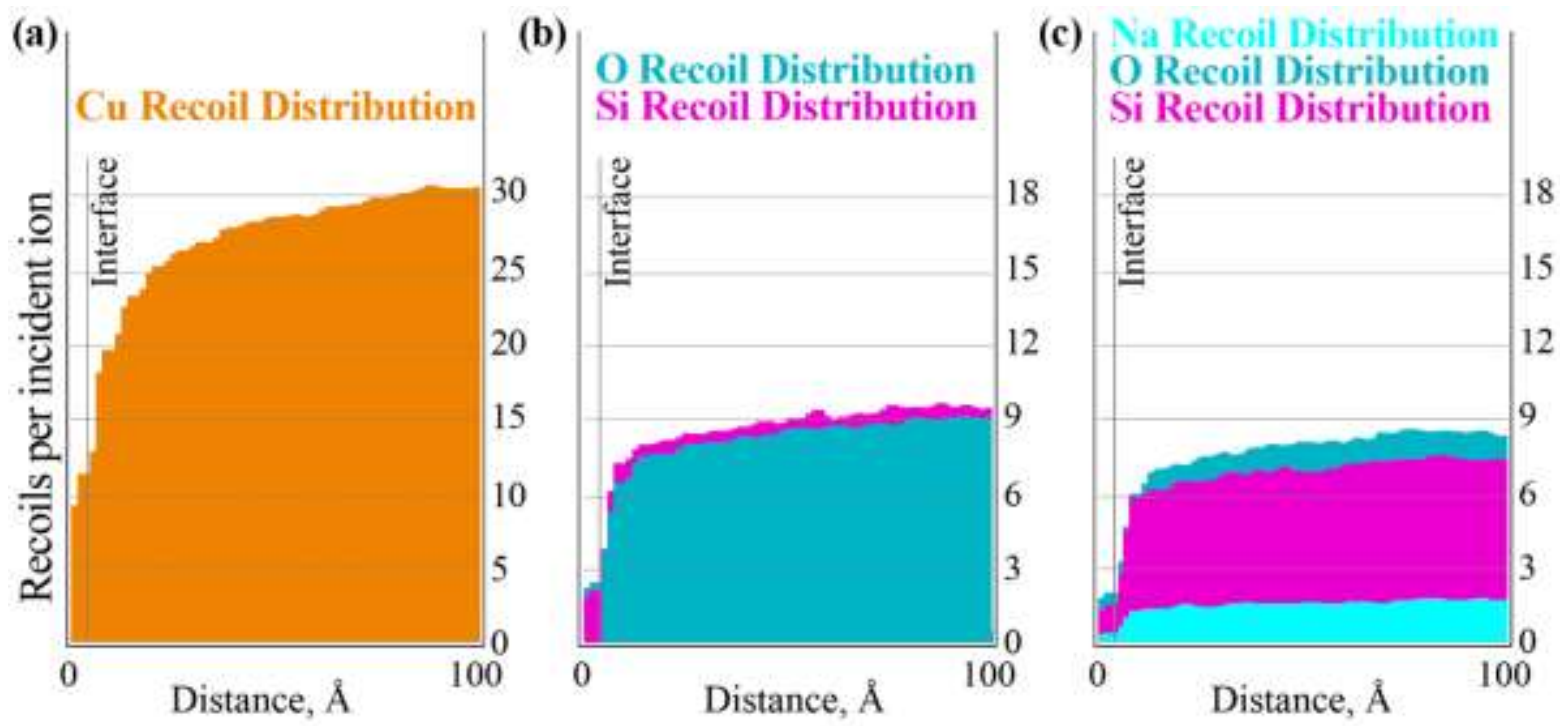

Figure 4. Statistical depth recoil distributions for graphene irradiated by xenon ions on (a) copper, (b) $\mathrm{SiO}_{2} / \mathrm{Si}$ and (c) glass substrates. For glass substrate, the distribution of other element atoms present in glass $(\mathrm{Ca}, \mathrm{Mg}, \mathrm{Al})$ is not shown due to its negligibly small rate.

According to our estimations, the approximate amount of displacements which can be created by recoil atoms in graphene layer was $6 \cdot 10^{9} \mathrm{~cm}^{-2}$ for copper, $4 \cdot 10^{9} \mathrm{~cm}^{-2}$ for $\mathrm{SiO}_{2} / \mathrm{Si}$ and 11 
$5 \cdot 10^{9} \mathrm{~cm}^{-2}$ for glass substrate, these values being more realistic than those estimated for substrate sputtering damage. Thus, we can consider recoil-dominated damage to be more intensive mechanism of defect formation in SHI-irradiated supported graphene than the substrate sputtering in our case.

Another reported mechanism to contribute to the defect formation in graphene irradiated by swift heavy ions is an electronically-stimulated surface desorption [23]. Due to a high energy of $\mathrm{Xe}$ ions used in our experiment, it can dissipate through an electronic excitation event, and thus the incident ions (or recoil atoms) can be considered as the hot electron source (that mostly relates to collisions with reduced direct kinetic energy transfer, i.e. non-central collisions with an impact outside the target atom cross section) [23]. In turn, the hot electrons disrupt graphene lattice, leading to the in-plane carbon bonds breakage [24]. According to our calculations, the electronic stopping energy loss in graphene for the case of $160 \mathrm{MeV}$ xenon ions is $17.2 \mathrm{keV} / \mathrm{nm}$, with this value being quite enough for the surface atoms to desorb. It should be noted that the $\mathrm{keV} / \mathrm{nm}$ units are used here for a comparative purpose only, as in [17]; the physical meaning of nanometers in the denominator is not defined for graphene and requires adaptation to be correctly used both for three- and two-dimensional targets.

Naturally, the substrate does not affect the amount of energy lost for ionization in graphene within the utilized approach; however, according to our calculations, electronic stopping energy loss for $160 \mathrm{MeV}$ xenon ions in copper is $36.3 \mathrm{keV} / \mathrm{nm}$ (it can be considered quantitative since the substrate is a bulk target). A large value of this parameter suggests an idea that the contribution to the defect formation in graphene is possible from substrate hot electrons produced near the interface. Due to silicon oxide giving the greatest contribution to electronic stopping in glass, the ionization energy loss for $\mathrm{SiO}_{2} / \mathrm{Si}$ and glass has almost similar values: 14.5 and $14.6 \mathrm{keV} / \mathrm{nm}$, respectively. Energy lost for ionization by recoil atoms turned out to be small (less than $2 \mathrm{eV}$ ), as well as the amount of recoil-induced ionization events for all three cases. 
It should be noted that the simulation performed did not take into account the fact that graphene on copper is as-grown, but it nevertheless agreed with the supporting Raman experiments qualitatively well for both pristine and irradiated graphene, leading to a conclusion that substrate-induced irradiation effects in case of a relatively strong graphene-metal interaction manifest themselves much stronger than the residual synthesis effects such as chemical bonds at the interface.

Finally, considering the possibility of doping for air-exposed graphene, we analyzed indicative in this case Raman spectra parameters ( $G$ and 2D peak positions, $I(2 D) / I(G)$ ratio [25]) for graphene on $\mathrm{SiO}_{2} / \mathrm{Si}$ substrate. As it was seen in Fig. 1, the evolution of these parameters is observed as the fluence increases, suggesting the increasing hole doping for greater defect density values [25]. Summarizing corresponding obtained values for each spectrum in a batch processing sequence, we obtained fluence-dependent average values of hole concentration presented in Fig. 5. A near-linear dependence on $\left\langle\mathrm{I}_{\mathrm{D}} / \mathrm{I}_{\mathrm{G}}\right\rangle$ is observed in this case, demonstrating that 'SHI irradiation + functionalization' sequence can be considered a comparable alternative to the low-energy ion beam implantation [26] for graphene doping. Peak ratio value corresponding to the initial defect density of pristine graphene which is not determined by the irradiation effects still fits into the obtained line well. This fact suggests that for graphene functionalization methods based on a defect induction through SHI irradiation, a small but present initial amount of defects represented by grain boundaries or vacancies does not strongly affect dependencies needed for controllability of the process. Presented observation can be taken into account in order to simplify graphene-based nanoelectronic device engineering process. 


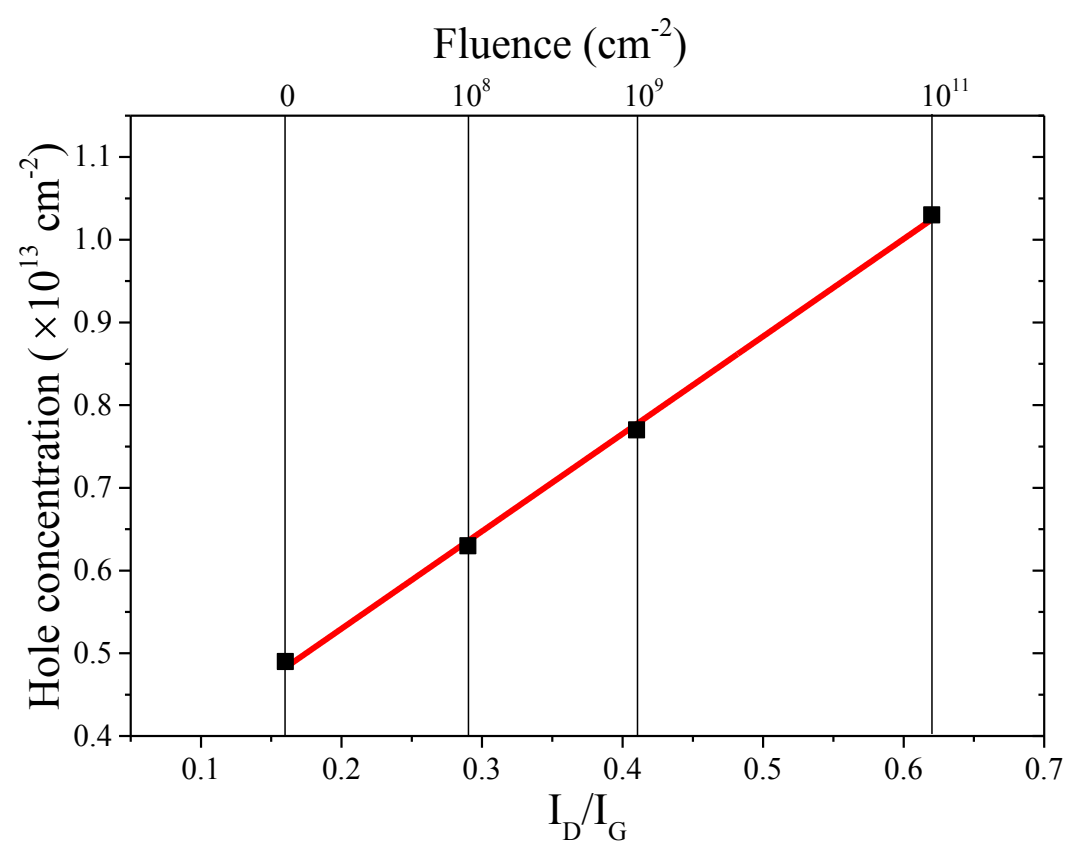

Figure 5. Hole concentration dependence on $\left\langle\mathrm{I}_{\mathrm{D}} / \mathrm{I}_{\mathrm{G}}>\right.$ ratio representing the average sample defect density (fluence also indicated) for graphene on $\mathrm{SiO}_{2} / \mathrm{Si}$ irradiated with $160 \mathrm{MeV} \mathrm{Xe}$ ions. Squares represent experimental data obtained from Raman spectra and solid line shows a linear fit.

\section{Conclusion}

A systematic Raman study, as well as corresponding Monte-Carlo simulations for supported graphene irradiated by swift xenon ions (energy of $160 \mathrm{MeV}$ ) were performed. As the fluence increased, changes in the defect density and dominating defect types were observed. In order to analyze the contributions of defect formation mechanisms in which the substrate is involved, a comparative study was performed for graphene on $\mathrm{SiO}_{2} / \mathrm{Si}$, copper and glass substrates. The contribution of sputtered substrate atoms was small for all cases, while the major defining mechanisms were atomic recoils reaching graphene-substrate interface and electronically-stimulated surface desorption. A role of substrate recoils reaching the interface was found to be greater for graphene on copper, while for $\mathrm{SiO}_{2} / \mathrm{Si}$ and glass substrates atom recoils participated approximately equally. Moreover, a possibility of defect formation in graphene due to substrate hot electrons, generated near the interface, was noted. Considering the possibility of doping for the air-exposed graphene, a linear hole concentration dependence on the $\left\langle\mathrm{I}_{\mathrm{D}} / \mathrm{I}_{\mathrm{G}}>\right.$ was found, with the initial defectiveness point (not defined by the irradiation effects) still 
fitting into the obtained dependence well. The present study provides further insight into the contributions of different defect formation mechanisms in irradiated graphene. The obtained results are useful for graphene-based nanoelectronic device engineering, including devices that require controlled graphene functionalization as well as controlled introduction of a known amount of disorder into graphene structure.

\section{References}

[1] A.C. Ferrari, D.M. Basko, Nature Nanotech. 8, 235-246 (2013). DOI: 10.1038/nnano.2013.46

[2] A.K. Geim, Science 324, 1530-1534 (2009). DOI: 10.1126/science.1158877.

[3] B. Guo, Q. Liu, E. Chen, H. Zhu, L. Fang, J.R. Gong, Nano Lett. 10, 4975-4980 (2010). DOI: $10.1021 / \mathrm{nl103079j}$.

[4] D.W. Boukhvalov and M.I. Katsnelson. Nano Lett. 8, 4373-4379 (2008). DOI: $10.1021 / n 1802234 n$.

[5] J. Zeng, H.J. Yao, S.X. Zhang, P.F. Zhai, J.L. Duan, Y.M. Sun, G.P. Li, J. Liu, Nucl. Instr. Meth. Phys. Res. B 330, 18-23 (2014). DOI: 10.1016/j.nimb.2014.03.019.

[6] S. Akcöltekin, H. Bukowska, T. Peters, O. Osmani, I. Monnet, I. Alzaher, B. Ban d'Etat, H. Lebius, M. Schleberger, Appl. Phys. Lett. 98, 103103 (2011). DOI: 10.1063/1.3559619.

[7] G. Compagnini, F. Giannazzo, S. Sonde, V. Raineri, E. Rimini, Carbon 47, 3201-3207 (2009). DOI: 10.1016/j.carbon.2009.07.033.

[8] S. Zhao, J. Xue, Y. Wang, S. Yan, Nanotechnology 23, 285703 (2012). DOI: 10.1088/0957$4484 / 23 / 28 / 285703$.

[9] S. Mathew, T.K. Chan, D. Zhan, K. Gopinadhan, A.-R. Barman, M.B.H. Breese, S. Dhar, Z.X. Shen, T. Venkatesan, J.T.L. Thong, Carbon 49, 1720-1726 (2011). DOI: 10.1016/j.carbon.2010.12.057.

[10] B.N. Gikal, S.N. Dmitriev, G.G. Gul'bekyan, P.Yu. Apel', V.V. Bashevoi, S.L. Bogomolov, O.N. Borisov, V.A. Buzmakov, I.A. Ivanenko, O.M. Ivanov, N.Yu. Kazarinov, I.V. Kolesov, V.I. Mironov, A.I. Papash, S.V. Pashchenko, V.A. Skuratov, A.V. Tikhomirov, M.V. Khabarov, 
A.P. Cherevatenko, N.Yu. Yazvitskii, Phys. Part. Nucl. Lett. 5, 33-48 (2008). DOI: $10.1134 / \mathrm{S} 1547477108010068$.

[11] J.F. Ziegler, M.D. Ziegler, J.P. Biersack, Nucl. Instr. Meth. Phys. Res. B 268, 1818-1823 (2010). DOI: 10.1016/j.nimb.2010.02.091.

[12] R. Giro, B. S. Archanjo, E. H. Martins Ferreira, R. B. Capaz, A. Jorio, C. A. Achete, Nucl. Instr. Meth. Phys. Res. B: Beam Interact. Mater. At. 319 (2014), 71-74. DOI: 10.1016/j.nimb.2013.10.028.

[13] A.V. Krasheninnikov and K. Nordlund. J. Appl. Phys. 107 (2010), 071301. DOI: $10.1063 / 1.3318261$.

[14] D.C. Bell, M.C. Lemme, L.A. Stern, J.R. Williams and C.M. Marcus. Nanotechnology 20 (2009), 455301. DOI: 10.1088/0957-4484/20/45/455301.

[15] L.G. Cançado, A. Jorio, E.H. Martins Ferreira, F. Stavale, C.A. Achete, R.B. Capaz, M.V.O. Moutinho, A. Lombardo, T.S. Kulmala, A.C. Ferrari, Nano Lett. 11, 3190-3196 (2011). DOI: $10.1021 / \mathrm{nl} 201432 \mathrm{~g}$.

[16] A. Eckmann, A. Felten, A. Mishchenko, L. Britnell, R. Krupke, K.S. Novoselov, C. Casiraghi, Nano Lett. 12, 3925-3930 (2012). DOI: 10.1021/nl300901a.

[17] W. Li, X. Wang, X. Zhang, S. Zhao, H. Duan \& J. Xue. Sci. Rep. 5 (2015), 9935. DOI: 10.1038/srep09935.

[18] O. Lehtinen, J. Kotakoski, A. V. Krasheninnikov, A. Tolvanen, K. Nordlund, and J. Keinonen. Phys. Rev. B 81 (2010), 153401. DOI: 10.1103/PhysRevB.81.153401.

[19] J. Zeng, J. Liu, H.J. Yao, P.F. Zhai, S.X. Zhang, H. Guo, P.P. Hu, J.L. Duan, D. Mo, M.D. Hou, Y.M. Sun. Carbon 100 (2016), 16. DOI: 10.1016/j.carbon.2015.12.101.

[20] S. Mathew, T.K. Chan, D. Zhan, K. Gopinadhan, A.R. Barman, M.B.H. Breese, S. Dhar, Z.X. Shen, T. Venkatesan, John TL Thong. Carbon 49 (2011), 1720-1726. DOI: 10.1016/j.carbon.2010.12.057. 
[21] P. Ahlberg, F.O.L. Johansson, Z.-B. Zhang, U. Jansson, S.-L. Zhang, A. Lindblad, and T. Nyberg. APL Materials 4 (2016), 046104. DOI: 10.1063/1.4945587.

[22] K. Yoon, A. Rahnamoun, J.L. Swett, V. Iberi, D.A. Cullen, I.V. Vlassiouk, A. Belianinov, S. Jesse, X. Sang, O.S. Ovchinnikova, A.J. Rondinone, R.R. Unocic, and A.C.T. van Duin. ACS Nano 10 (2016), 8376-8384. DOI: 10.1021/acsnano.6b03036.

[23] A.V. Krasheninnikov, Y. Miyamoto, D. Tománek. Phys. Rev. Lett. 99 (2007), 016104. DOI: 10.1103/PhysRevLett.99.016104.

[24] M. Lenner, A. Kaplan, Ch. Huchon, and R. E. Palmer. Phys. Rev. B 79 (2009), 184105. DOI: 10.1103/PhysRevB.79.184105.

[25] R. Beams, L. Gustavo Cançado, L. Novotny. J. Phys. Condens. Matter 27 (2015), 083002. DOI: $10.1088 / 0953-8984 / 27 / 8 / 083002$.

[26] P. Willke, J.A. Amani, A. Sinterhauf, S. Thakur, T. Kotzott, T. Druga, S. Weikert, K. Maiti, H. Hofsäss, and M. Wenderoth. Nano Lett. 15 (2015), 5110-5115. DOI: 10.1021/acs.nanolett.5b01280. 